\title{
Embryonendebatte auf verfassungsrechtlichen Abwegen?
}

\section{A. LuftSiG, Folter, Embryonenschutz zwischen Verfassungsrecht und Philosophie:}

Embryonen stellen sozusagen das Stadium zwischen geborenen Menschen und künftigen Generationen dar. Der Umgang mit ihnen, der anders als in den Flugzeugabschußund den Folterkonstellationen - in denen ebenfalls seit längerem über gesellschaftliche Grundwerte in Gestalt von Menschenwürde und Lebensschutz verhandelt wird auch nicht nur extreme Ausnahmefälle betrifft, führt jedoch in fundamentale Konflikte. Es geht um Abtreibung zur Garantie der freien Persönlichkeitsentfaltung für die Mütter, noch mehr geht es heute aber um Präimplantationsdiagnostik (PID) und Früheuthanasie zur Verhinderung erbkranker Kinder sowie um eine (verbrauchende) Embryonenforschung mit dem Ziel der Gewinnung medizinischer Erkenntnisse (oder gar der Züchtung von Ersatzzellen oder Organen). Die gentechnische Entwicklung ist hier seit längerem dabei, eine technologische Revolution auszulösen. Dies birgt einerseits u.U. erhebliche medizinische und wirtschaftliche Chancen, z.B. in der perspektivischen Heilung Schwerkranker, aber auch in puncto Selbstbestimmungsrecht der (etwaigen) Eltern. Und diese Chancen können nur wahrgenommen werden, wenn man menschliche Stammzellen zu Forschungszwecken einsetzt - und die Embryonen so letzten Endes »tötet«. Andererseits entsteht eine prinzipielle Infragestellung unseres Selbstverständnisses als autonome Wesen, wenn demnächst vielleicht die Eltern darüber entscheiden können, mit welchem Gen-Set jemand zur Welt kommt. Während darum den einen die genannten Praktiken durch die Menschenwürde untersagt zu sein scheinen, wenn doch scheinbar Embryonen nicht mehr »als Personen respektiert « werden, treten andere für eine weitgehende Erlaubnis der Praktiken ein - sei es um der Selbstbestimmung der Mütter willen, sei es wegen des medizinischen Fortschritts, der mittelfristig verbesserte Heilungschancen für Schwerkranke verheißt. Damit nimmt die Embryonendebatte wie auch die jüngsten Kontroversen zum LuftSiG und zur Folter, zentral auf Würde und Lebensschutz Bezug.

Insbesondere dort, wo die Menschenwürde betroffen ist, führt eine »rein verfassungsrechtliche « Betrachtung anhand juristischer Auslegungsmethoden oftmals zu nur unzureichenden Ergebnissen. Denn der Begriff entstammt genuin der Philosophie und ist auch vom reinen Wortsinn her wenig klar. Gleichzeitig ist er der Basisbegriff liberaler Verfassungen; er muß daher geklärt werden, kann aber womöglich nur geklärt

1 Der erstgenannte Verfasser lehrt an der Universität Bremen europäisches und deutsches Umwelt- und Verfassungsrecht sowie Gerechtigkeits- und Steuerungstheorie. Der zweitgenannte Autor bearbeitet ein Forschungsprojekt zur roten und grünen Gentechnik an jener Professur. Vgl. als Hintergrund dieses Beitrags auch die Habilschrift von Ekardt (2004), Zukunft in Freiheit: Eine Theorie der Gerechtigkeit, der Grundrechte und der politischen Steuerung - zugleich eine Grundlegung der Nachhaltigkeit, §§ 3, 5 B. sowie dazu die präzisierte und ergänzte C.H.Beck-Taschenbuchfassung Ekardt (2005), Das Prinzip Nachhaltigkeit: Generationengerechtigkeit und globale Gerechtigkeit, Kap. II, III, IV, V A. 
werden, wenn man nach seiner Begründung fragt. Und diese normative Begründung kann, da Würde verfassungsrechtlich selbst der »grundlegendste« Begriff ist, nicht mehr selbst in der Verfassung gesucht werden. Dies wirft zugleich ein Schlaglicht darauf, daß die Rechtsfindung durch Gesetze und im Einzelfall eine Art Sonderfall der allgemeinen normativen (moralischen) Debatte ist und nicht etwa deren empirisches Gegenstück $^{2}$ darstellt. Die Findung und Interpretation von Recht hat auch schon deswegen nicht den Charakter einer »Tatsachenerhebung «, weil das Ergebnis der Interpretation einen Aufforderungscharakter hat und damit eine Wertung beinhaltet, anders als Tatsachen, und zudem durch die stets zu prüfende Möglichkeit verfassungskonformer Auslegungen sowie durch die teleologische Auslegung noch die einfachste Norminterpretation nicht nach Art eines empirischen Faktums einfach »beobachtet« werden kann. »Wertend « meint bei alledem stets »auf das (Handeln-)Sollen bezogen«, figuriert also nicht als Synonym für »subjektiv« oder gar »beliebig«.

Im vorliegenden Beitrag soll es nicht um die vielfältig dargestellten naturwissenschaftlichen Grundlagen der Embryonendebatte, um die Einzelheiten der bisher bestehenden einfachgesetzlichen Verbotsnormen oder auch um eine Verbotsnorm wie Art. 3 EuGRC - mit Verboten für reproduktives Klonen, Erzeugung von Embryonen zu Forschungszwecken, eugenische Praktiken usw. - bis hin zur Einzelbeurteilung der verschiedenen technischen Verfahren gehen ${ }^{3}$, sondern um die Frage, inwieweit aus bestimmten fundamentalen Wertungen liberaler Verfassungen Aussagen über den Status des Embryos in vitro gewonnen werden können. Das BVerfG glaubt dies offenbar zu können; der EGMR z.B. glaubt es dagegen wohl nicht. Unsere These ist demgegenüber, daß es einer verfassungsinterpretativen Neupositionierung jenseits der beiden bisherigen Hauptströmungen bedarf, mit denen im folgenden eine Auseinandersetzung stattfinden soll. In der Sache ist dies - angesichts unseres Versuchs, die Menschenwürde anders als bisher zu interpretieren - zugleich eine Kritik an anderen BVerfGEntscheidungen wie etwa das im Februar 2006 verkündete Urteil über das Luftsicherheitsgesetz. ${ }^{4}$

2 Dies wird durch die beliebte, aber wenig reflektierte Forderung nach einer strengen »Trennung von Recht und Moral « verdeckt. Richtigerweise kann - vor dem Hintergrund der eben gemachten Ausführungen - jene Trennung nur etwas anderes meinen: nämlich eine Trennung von Gerechtigkeit und gutem Leben (also die Scheidung der potentiell universalen Moralnormen einschließlich ihrer Konkretisierung durch demokratische Mehrheiten einerseits und der Sitten und Gebräuche, die man privat oder in einer Gruppe pflegen mag, für die sich aber keine universalistischen Gründe anführen lassen und die zudem nur schwer in Rechtssätze überführbar sind. - All dies ändert übrigens nichts daran, daß der moralische Diskurs im Recht jenseits der Klärung der juristischen Grundprinzipien in der Diskussion über verfassungskonforme Auslegungen bzw. Verfassungskonformitätsfragen aufgehen muß, um Rechtssicherheit zu erwerben.

3 Vgl. zu alledem Kornack (2006), Embryonenforschung und »Früheuthanasie«, in: Ekardt (Hg.), Generationengerechtigkeit und Zukunftsfähigkeit, S. 260 ff. m.w.N.

4 Vgl. dazu Ekardt (2006), Frankfurter Rundschau vom 18.02., S. 9; zur Folter Ekardt (2006a), Neue Justiz, S. 64 ff. (wobei in beiden Fällen wie auch in diesem Aufsatz ein weitgehendes, aber nicht absolutes Verbot begründet wird - mit den selben Argumenten wie vorliegend). 


\section{B. Verfassungsrechtliche Position 1 - absoluter Schutz des Embryo in vitro (zugleich eine Kritik am BVerfG):}

Im wesentlichen gibt es in der juristischen Diskussion zwei Grundpositionen: die herrschende Meinung sieht den Embryo ab der Verschmelzung von Ei- und Samenzelle als Menschen an, der somit den Schutz der Menschenwürde - entweder objektiv- oder subjektiv-rechtlich -genieße. Daraus wird sodann aufgrund der ebenfalls nach h.M. bestehenden Unabwägbarkeit der Menschenwürde eine Abwägung mit anderen Interessen für unzulässig erklärt. (Der Einfachheit der Darstellung wegen sollen an dieser Stelle zwei vergleichbare Ansichten zusammengefaßt werden: eine, nach der der Embryo in vitro Träger des Prinzips Menschenwürde gemäß Art. 1 Abs. 1 GG - welches das begründende Prinzip der Grundrechte ist, ob man das Prinzip nun selbst ebenfalls als Grundrecht liest oder auch nicht - ist und eine zweite, die ihn als Träger des Lebensrechts gemäß Art. 2 Abs. 2 S. 1 GG betrachtet, wobei aber eine gesetzliche Beschränkung gemäß Art. 2 Abs. 2 S. 3 im Bereich der Embryonenforschung nicht möglich sei, da sie eine Verletzung der Menschenwürde darstelle, welche aber einer Abwägung gerade unzugänglich sei. ${ }^{5}$ Entscheidend ist bei beiden Positionen, daß der Embryos selbst Grundrechtsträger ist und eine Abwägung mit kollidierenden Belangen ausgeschlossen wird. ${ }^{6}$ ) Die Gegenposition kommt aufgrund unterschiedlicher möglicher Ansätze zu einer Abwägung zwischen den Belangen des in vitro fertilisierten (ivf) Embryo und den widerstreitenden Belangen, insbesondere den Grundrechten der Forschungsfreiheit sowie derjenigen, die gesundheitlich von den Ergebnissen der u.U. für die Medizin entscheidenden Embryonenforschung profitieren könnten - so z.B. Träger von Erbkrankheiten. Dabei wird dem Embryo der Status des Grundrechtsträgers teils auch gänzlich bestritten.

Schon bei der Bestimmung des Schutzbereiches der Menschenwürde ergeben sich erhebliche Schwierigkeiten. So erklärt Art. 1 Abs. 1 S. 1 GG schlicht: »Die Würde des Menschen ist unantastbar.«Was Würde genau heißt, erfährt man dort jedoch nicht. Es hat in der Vergangenheit zwar zahlreiche Versuche gegeben, den Umfang der Menschenwürde positiv oder negativ zu bestimmen. Ein eindeutig anwendbares Ergebnis ist die bisherige juristische Diskussion dabei jedoch schuldig geblieben. Im Bereich der positiven Definition der Menschenwürde sind im wesentlichen zwei Ansatzpunkte auszumachen ${ }^{7}$ : nach der Mitgiftlehre ist Würde ein von Gott gegebener bzw. in der menschlichen Vernunft und sittlichen Autonomie wurzelnder (Eigen-)Wert des Menschen. ${ }^{8}$ Diese Theorie hat nun aber Schwierigkeiten, ihren Ansatz zu begründen, ohne

5 Vgl. dazu auch die Hinweise bei BVerfGE 75, 369 (380); 93, 266 (293).

6 Für die Gleichsetzung des Würdeschutzes und des Lebensrechts Höfling (2003), in: Sachs (Hg.), Grundgesetz-Kommentar, 3. Aufl., Art. 1 Rn. 49 m.w.N.; dagegen Schulze-Fielitz (2004), in: Dreier (Hg.), Grundgesetz-Kommentar, 2. Aufl., Art. 2 II Rn. 27.

7 Vgl. zur Übersicht auch über weitere Konzepte Augustin (2004), Argumentationsmuster: Menschenwürde im Zusammenspiel von Recht und Philosophie, in: Stoecker (Hg.), Menschenwürde. Annäherung an einen Begriff, S. $111 \mathrm{ff}$.

8 Vgl. Dreier (2004), in: Dreier (Hg.), Grundgesetz-Kommentar, 2. Aufl., Art. 1 I Rn. 55 m.w.N.; zur geschichtlichen Entwicklung Giwer (2001), Rechtsfragen der Präimplantationsdiagnostik, S. 136 f. m.w.N. 
dabei auf vordefinierte Werte zurückzugreifen. Entweder werden hier die Existenz Gottes oder bestimmte Werte (in platonischer Tradition) per se vorausgesetzt - oder der menschlichen Vernunft wird ein bestimmter »Inhalt « zugeschrieben, ohne daß deutlich wird, wie dieser hergeleitet werden kann. ${ }^{9}$ Dieser Schwierigkeit entgeht die häufig mit Habermas und Luhmann in Verbindung gebrachte Leistungslehre, indem sie Würde nicht als von Anfang an existent annimmt, sondern einen individuellen Prozeß der Identitätsbildung zur Erlangung von Würde verlangt. Hierbei werden allerdings möglicherweise Menschen, die der Identitätsbildung noch nicht - was gerade für die Embryonenthematik relevant wäre - oder nicht mehr fähig sind, vom Schutzbereich ausgenommen; daher wird sie vom BVerfG als deutschem Verfassungsgericht bisher stets zurückgewiesen. ${ }^{10}$ Die herrschende Meinung im juristischen Diskurs hierzulande versucht den so explizierten Schwierigkeiten zu entgehen, indem sie die Menschenwürde ergänzend nicht über ihren Umfang, sondern mehr über die mögliche Verletzungshandlung definiert. Hierzu greift sie auf die von Dürig entwickelte und auf eine Kant-Formulierung anspielende Objekt-Formel zurück, die auch das BVerfG teilweise aufgegriffen hat. Danach liegt eine Verletzung der Menschenwürde jedenfalls dann vor, wenn man einen Menschen als bloßes Mittel gebraucht ${ }^{11}$ - wobei hierzu die Subjektqualität des Betroffenen grundsätzlich in Frage gestellt, also der jedem Menschen um seiner selbst willen zukommende Wert mißachtet sein muß. ${ }^{12}$ Mit dem Vorwurf der Beliebigkeit wird diese Formel jedoch schon innerhalb des gängigen juristischen Diskurses vielfach kritisiert bzw. als unbegründbar abgelehnt ${ }^{13}$, teilweise sogar die Menschenwürde an sich als »inhaltslose Tautologie « ${ }^{14}$ für normativ irrelevant erklärt. Kaum bestreitbar dürfte hier jedenfalls sein, daß das bloße Verbot einer irgendwie gearteten Instrumentalisierung anderer Menschen nicht der normative Gehalt des Würdeprinzips sein könnte. Andernfalls wären große Teile menschlichen Handelns, welches nahezu immer auch instrumentell rational (also auf die Verwirklichung eigennütziger Ziele, wobei die anderen als Mittel zur Zielverwirklichung erscheinen) geprägt ist, offenbar verfassungswidrig.

9 Zur diesbezüglichen Kritik auch Ekardt (2005), Kap. II A. und IV A.

10 BVerfGE 87, 209 (228); Starck (2005), in: v. Mangoldt/ Klein/ Starck (Hg.), Kommentar zum Grundgesetz , 5. Aufl., Art. 1 Abs. 1 Rn. 18.

11 Dürig, AöR 81 (1956), 117 (127); die ursprüngliche, komplexere Formulierung findet sich bei Kant (1963), Grundlegung zur Metaphysik der Sitten, in: Gesammelte Werke, hrsg. von Weischedel, Bd. VIII, S. 600 - hier zitiert nach Hoerster (2002), Ethik des Embryonenschutzes, S. 13.

12 Vgl. BVerfG, NJW 2004, 999 (1001 f.).

13 Siehe etwa Dreier (2004), Art. 1 I Rn. 53 m.w.N.; Pieroth/ Schlink (2004), Grundrechte Staatsrecht II, 20. Aufl., Rn. 360.

14 Hoerster (2002), S. 20; dagegen Kardinal Lehmann (2003), Ethik am Lebensbeginn, S. 16. Dies versucht wiederum Herdegen (2005), Gedächtnisschrift Heinze, S. 361 ff. durch eine »wertende Gesamtbetrachtung « unter Berücksichtigung des geschichtlichen Erbes, dem Vergleich mit anderen rechtsstaatlichen Ordnungen und »einem Mindestmaß an Evidenz der Würdeverletzung « zu vermeiden. Doch kann man wiederum von einer »evidenten Verletzung « einer Norm erst sprechen, wenn zunächst einmal klar ist, was die Norm überhaupt bedeutet. Außerdem ist der Vorschlag auch deshalb problematisch, weil näher geklärt werden müßte, inwieweit vormalige (womöglich wenig demokratische) oder ausländische Rechtsordnungen einen Auslegungsgesichtspunkt für die Verfassung abgeben können. 
Nach dieser Grundlegung stellt sich die Frage nach dem Status des Embryos in vitro. Entstehungsgeschichtlich gab es während der Beratungen des Parlamentarischen Rates einen Antrag der Fraktion der seinerzeitigen Deutschen Partei auf ausdrückliche Miteinbeziehung des »keimenden Lebens « in Art. 2 Abs. 2 GG. Dieser wurde jedoch mehrheitlich abgelehnt. Umstritten ist jedoch der Grund für diese Ablehnung. Aus dem Schlußbericht des Berichterstatters, der allerdings einem Widerspruch im Plenum nicht zugänglich war und darum nichts aussagen muß, geht hervor, der Ausschuß sei davon ausgegangen, auch das »keimende Leben « sei bereits vom Recht auf Leben erfaßt, eine Klarstellung daher unnötig, wohingegen vor der Abstimmung über den Antrag ein Abgeordneter wohl für die Mehrheit sich gegen eine Ausweitung des Rechts auf Leben ausgesprochen hatte. Insofern ist entstehungsgeschichtlich der Gehalt des Art. 2 Abs. 2 GG (und auch des Art. 1 GG) jedenfalls unklar. ${ }^{15}$

Das BVerfG kommt dennoch unter Heranziehung grammatischer und systematischer Verfassungsauslegungsmethoden zu einem klaren Ergebnis hinsichtlich des Schutzes vorgeburtlichen Lebens. So stellt das Gericht zum Schwangerschaftsabbruch ${ }^{16}$ fest: »Leben im Sinne der geschichtlichen Existenz eines menschlichen Individuums besteht nach gesicherter biologisch-physiologischer Erkenntnis jedenfalls vom 14. Tage nach der Empfängnis (Nidation, Individuation) an «. ${ }^{17}$ Zumindest ab diesem Zeitpunkt entwickele sich das Leben »nicht erst zum Menschen, sondern als Mensch $\ll{ }^{18}$ Es führt weiterhin aus, medizinische Erkenntnisse legten nahe, das Leben beginne gar schon mit der Verschmelzung von Ei- und Samenzelle, läßt eine rechtliche Bewertung aber explizit offen. ${ }^{19}$ In der gängigen Literatur wird eben dieser Zeitpunkt der Befruchtung im biologischen Sinne meist als Beginn des menschlichen Lebens angenommen. ${ }^{20}$ Nicht ganz klar erscheint jedoch nicht nur die Begründung, sondern auch die Folgerung aus diesem Befund: ebenso wie das BVerfG fordert eine Vielzahl von Autoren, »wo menschliches Leben existiert, kommt ihm Menschenwürde zu«, unabhängig von Faktoren wie Schmerzempfindlichkeit oder sozialer Interaktion. ${ }^{21}$ Neben dieser »naturwissenschaftlichen« Begründung des Schutzstatus des Embryos wird das Kontinuitätsargument vorgebracht: Es finde ab der Befruchtung ein

15 Vgl. Schulze-Fielitz (2004), Art. 2 II Rn. 5.

16 BVerfGE 39, 1 (Fristenlösung I); 88, 203 (Fristenlösung II).

17 BVerfGE 39, 1 (37).

18 BVerfGE 88, 203 (252).

19 Siehe BVerfGE 88, 203 (251).

20 Vgl. Herdegen (2003), in: Maunz/ Dürig (Hg.), Grundgesetz-Kommentar, Bd. 1, Art. 1 Abs. 1, Rn. 61; Jarass (2004), in: Jarass/ Pieroth, Grundgesetz-Kommentar, 7. Aufl., Art. 1 Rn. 6; Murswiek (2003), in: Sachs (Hg.), Grundgesetz-Kommentar, Art. 2 Rn. 143; Schulze-Fielitz (2004), Art. 2 II Rn. 29; Starck (1999), Art. 2 Abs. 2, Rn. 176; Böckenförde (2003), Juristenzeitung, S. 809 (812); Kloepfer (2002), Juristenzeitung, S. 417 (420); offen Ipsen (2001), Juristenzeitung, S. 989 (994).

21 BVerfGE 39, 1 (41); 88, 203 (252); Höfling (2003), Art. 1 Rn. 49 ff.; Starck (1999), Art. 1 Abs. 1 Rn. 17 f.; Jox (2002), Euthanasie und Embryonenforschung, in: Nicht/ Wildfeuer (Hg.), Person - Menschenwürde - Menschenrechte im Disput, S. 288; Eibach (2003), Medizinrecht, S. 441 (445); Faßbender (2003), Medizinrecht, S. 279 (281); Wiebe (2004), Zeitschrift für Lebensrecht, S. 9 (10). 
gleichmäßiger Prozeß der Entwicklung statt, dessen Aufspaltung in einzelne Phasen willkürlich sei, weswegen ab der Befruchtung der Würde- bzw. Lebensschutz greifen müsse. ${ }^{22}$ Jenes Kontinuitätsargument ist indes nicht haltbar, da es sich hier um einen Fall des seit der Antike bekannten Haufen-Paradox handelt. Hiernach kann es auch bei einem kontinuierlichen Prozeß Gründe geben, diesen in unterschiedliche Abschnitte einzuteilen. ${ }^{23}$ Beispiel: Man kann zwar nicht sagen, bei wieviel Sandkörnern der »Haufen« aufhört und das »Häufchen« beginnt. Trotzdem ist die Scheidung von Haufen und Häufchen ersichtlich nicht sinnlos.

Ebenso ist auch das »naturwissenschaftliche « Argument zweifelhaft. Viele sehen in diesem Argument einen Schluß von naturwissenschaftlichen Daten auf die Zuerkennung der Würde und des Lebensrechts - und damit einen naturalistischen Fehlschluß. ${ }^{24}$ Zumindest erscheint das Argument zirkulär; denn es ist doch allererst die Frage, wer ein »jeder « und ein »Mensch« im Sinne des Grundrechtskatalogs ist, der dann als Träger von Würde und Lebensrecht anzusehen wäre. ${ }^{25}$ Weiterhin führt eine angenommene Subjektqualität des Embryos und der volle Schutz von Lebensrecht und Menschenwürde ab dem Zeitpunkt der Verschmelzung von Ei- und Samenzelle zu einem Wertungswiderspruch: so stehe der Embryo in vitro unter dem unantastbaren Schutz der Menschenwürde, wohingegen der Embryo in utero abgetrieben bzw. in vivo dessen natürliche Nidation durch Maßnahmen, wie z.B. die Postkonzeptionspille (»Pille danach «) oder die Intrauterinalspirale, verhindert werden dürfe, was in beiden Fällen eine Vernichtung seiner Existenz bedeute. ${ }^{26}$ Für den Fall der Abtreibung im Rahmen der $\S \S 218,218$ a StGB wird dem aber wiederum entgegengehalten, hier bestehe eine existenzbegründende, womöglich sogar ungewollte Nähebeziehung zwischen der Frau und dem Embryo. Dies sei aber bei der gewollten IVF gerade nicht der Fall, die vielmehr planmäßig herbeigeführt werde, so daß es hier an einem entsprechend starken widerstreitenden Schutzgut mangele. ${ }^{27}$ Zudem sei im Intimbereich »Verhütung « weder ein staatliches Regulieren noch beweistechnisch eine Unterschei-

22 BVerfGE 39, 1 (37); Kardinal Lehmann (2003), S. 8 ff. m.w.N.; zu den biologischen Vorgängen vgl. auch Rager (2004), Zeitschrift für Lebensrecht, S. 66 (68 f.).

23 Vgl. Merkel (2002), Forschungsobjekt Embryo. Verfassungsrechtliche und ethische Grundlagen der Forschung an menschlichen embryonalen Stammzellen, S. 157 f. m.w.N.; Ekardt (2005), Kap. III C.; gegen die Anwendbarkeit des Haufen-Paradox auf das Kontinuitätsargument bei Embryonen Illies (2003), Das so genannte Potentialitätsargument am Beispiel des therapeutischen Klonens, in: Goebel/ Kruip (Hg.), Gentechnologie und die Zukunft der Menschenwürde, S. $34 \mathrm{f}$.

24 Vgl. Merkel (2001), Rechte für Embryonen?, Die Zeit Nr. 5; Dreier (2004), Art. 1 I Rn. 66 m.w.N.

25 Man kann hier auch nicht erwidern: Mensch im Sinne der Grundrechte sei eben jedes Lebewesen, das individuell der Gattung Mensch angehöre - denn es ist doch gerade erst die begründungsbedürftige These der h.M., daß dem so ist.

26 Vgl. zu diesen Argumenten Ipsen (2001), S. 989 (992); Ipsen (2004), Neue Juristische Wochenschrift, S. 268; Schlink (2003), Die überforderte Menschenwürde, Der Spiegel Nr. 51, 51 f.; Nationaler Ethikrat (2001), Stellungnahme zum Import menschlicher embryonaler Stammzellen, http://www.nationalerethikrat.de/stellungnahmen/pdf/Stellungnahme_ Stammzellimport.pdf [Stand: 08. Februar 2006], S. 19.

27 Vgl. Mildenberger (2002), Medizinrecht, S. 293 (298). 
dung zwischen natürlichen und künstlich bewirkten Abgängen möglich. ${ }^{28}$ Allerdings kann durch diese durchaus einleuchtenden praktischen Überlegungen nicht begründet werden, warum es dem Staat nicht geboten wäre, Werbung oder Verkauf von Nidationshemmern zu verbieten. ${ }^{29}$ Insofern besteht jedenfalls in diesem Bereich eine interne Inkonsistenz (von der generellen Frage nach der Begründungskraft »naturwissenschaftlicher « und zirkulärer Bestimmungen der Grundrechtsträgerschaft ganz abgesehen).

Besonders Merkel verweist darauf, daß die beiden Abtreibungsentscheidungen des BVerfG in diesem Kontext - unabhängig von der Frage nach der Begründungskraft eines bloßen Autoritätsarguments - keine Schlüsse auf einen eventuellen Schutz des Embryos durch die Menschenwürde erlaubten. Dies begründet er zu Recht mit der bereits angedeuteten logischen Widersprüchlichkeit der Entscheidungen. So postuliere das Gericht einerseits »Menschenwürde kommt schon dem ungeborenen menschlichen Leben $\mathrm{zu}$ «, »wo menschliches Leben existiert, kommt ihm Menschenwürde zu « und erklärt konsequent den Schwangerschaftsabbruch für »Unrecht ... und demgemäß rechtlich verboten«, bestätigt sodann jedoch die staatliche Verpflichtung zur Bereitstellung von ambulanten und stationären Einrichtungen für die Vornahme von Schwangerschaftsabbrüchen. ${ }^{30}$ Merkel sieht dies als »selbstdestruktiven Grundwiderspruch« einer »Rechtspflicht (des Staates, Anm. d. Verf.) zum Unrecht«, die schon aus normenlogischen Gründen unmöglich sei. ${ }^{31}$ Der Widerspruch liege darin, daß verfassungsrechtlich geschütztes »unschuldiges« Leben (und die Menschenwürde) abwägungsfest geschützt sei, das BVerfG aber sodann doch gerade selbst eine Abwägung zulasse. Hieraus folgert er, das Prinzip des lex posterior derogat legi priori anwendend: Die Feststellungen der zweiten Abtreibungsentscheidung bezüglich der staatlichen Einrichtungen zum Schwangerschaftsabbruch, welche neben der Bestätigung der ersten Entscheidung die »Teilnahmepflicht « des Staates am Schwangerschaftsabbruch begründeten, verdrängten das Postulat der Rechtswidrigkeit des Schwangerschaftsabbruchs und der Grundrechtssubjektivität des Embryos im allgemeinen. Dies unterstreicht er mit der Feststellung, eine nur postulierte Rechtsnorm, die trotz des Vorliegens ihrer Tatbestandsvoraussetzungen niemals Anwendung fände, stelle in Wirklichkeit keine Norm dar.

Merkel ist sicherlich zuzugestehen, daß die einschlägigen Entscheidungen des BVerfG in sich widersprüchlich sind. Allerdings scheint es begründungstechnisch fragwürdig, die Gültigkeit von Normen, insbesondere von Verfassungsrecht, davon abhängig zu machen, welche Teilaspekte von BVerfG-Entscheidungen die einfachen Gerichte anwenden und somit allein als geltend behandeln. Über die Begründung/Gerechtigkeit der Entscheidung sagt dies doch letztlich nichts, will man nicht behaupten, daß die Gerichte (was wohl kaum der Fall ist) inhaltlich beliebige Entscheidungen

28 In diesem Sinne etwa Schockenhoff (2003), Die Ethik des Heilens und die Menschenwürde. Moralische Argumente für und wider die embryonale Stammzellforschung, in: Robertson (Hg.), Der Perfekte Mensch, S. 104.

29 Vgl. Dreier (2002), Zeitschrift für Rechtspolitik, S. 377 (379); Merkel (2002), S. 61 f.

30 BVerfGE 88, 203 (328 ff.).

31 Hierzu und zum folgenden Merkel (2002), S. 72, 76 und $77 \mathrm{ff}$. 
treffen können. Art. 1, 2 GG sind nun einmal vorhanden und müssen interpretiert werden - was nicht damit zurückgewiesen werden kann, daß das BVerfG diese Normen falsch ausgelegt habe. Allerdings legt Merkel auch richtig dar, daß die Leitsätze einer Entscheidung ohne ihre Begründung nicht bestehen können. Insofern sind die Ausführungen des BVerfG aus diesen beiden Entscheidungen schwerlich argumentativ in die Diskussion um den Status des Embryos miteinzubeziehen.

Teilweise als Reaktion auf die Behauptung eines Potentials des Embryos ab der Kernverschmelzung wird eine besondere Situation beim Embryo in vitro insofern gesehen, als die Entwicklung desselben nicht auf natürlichem Wege erfolgen könne, sondern vielmehr ein weiteres Eingreifen, nämlich die Implantation des Embryos in den Uterus, nötig sei. ${ }^{32}$ Dies gelte um so mehr für bereits existente »überzählige « Embryonen. Diese entstehen, da wegen der immer noch geringen Erfolgsrate von in-vitro-Fertilisationen der Frau, die eine solche Befruchtung wünscht, mehrere Eizellen entnommen werden, um nicht später einen weiteren (risikobehafteten) Eingriff durchführen zu müssen. Weiterhin können »überzählige« Embryonen entstehen, wenn aus faktischen Gründen, z.B. wegen eines temporären oder andauernden Gesundheitszustands der Mutter, der die Implantation verbietet, oder gar wegen deren Tod eine Implantation nicht stattfinden kann. ${ }^{33}$ So man nicht die in-vitro-Fertilisation an sich verbietet, entstehen also zwangsläufig, wenn auch in geringem Maße, überzählige Embryonen. ${ }^{34}$ Bei diesen liege jedoch keinesfalls aktuelle Potentialität der Entwicklung hin zum geborenen Menschen vor, solange sie nicht in einen Uterus eingepflanzt werden. ${ }^{35}$ Nun wird zwar teilweise angenommen, für solche »überzähligen« Embryonen müsse es eine Art Implantationspflicht ${ }^{36}$ geben, um sie auf diese Weise zum Menschen werden zu lassen. Allerdings ist diesbezüglich nicht nur die Begründung unklar. Es ist ebenso unklar, wie eine Implantationspflicht überhaupt Sinn haben soll, wenn der Mutter gleichzeitig nach der Nidation eine Abtreibung erlaubt ist, wie dies bisher der Fall ist. Im übrigen erscheint es kaum als möglich, eine solche Pflicht mit der (unbestreitbar bestehenden) Menschenwürde sowie den Freiheitsrechten der Mütter oder gar fremder Frauen in Einklang zu bringen.

Ebenfalls untauglich ist der Versuch, die Erstreckung von Würde und Grundrechten daraus herzuleiten, daß die Würde gemäß Art. 1 Abs. 1 GG »unantastbar« sei und dies dann auch einen wirksamen Embryonenschutz erfordere. Denn dieser Begründungsversuch ist wiederum zirkulär: Es ist doch gerade erst die Frage, ob der Embryo ein »Mensch« im Sinne des Art. 1 GG ist! Einige der damit umrissenen Probleme kann man beispielhaft an folgender Formulierung von Otto exemplifizieren: »Der in der Würde begründete Rechtsstatus verweist auf die einfache Erkenntnis: Der Mensch ist als Mensch

32 Herdegen (2003), Art. 1 Abs 1, Rn. 62; Ipsen (2004), S. 268 (269).

33 Vgl. Illiadou (1999), Forschungsfreiheit und Embryonenschutz, S. 27 f. m.w.N.

34 Vgl. Enquête-Kommission »Recht und Ethik der modernen Medizin« (2002), in: Deutscher Bundestag Referat Öffentlichkeitsarbeit (Hg.), Stammzellforschung und die Debatte des Deutschen Bundestages zum Import von menschlichen embryonalen Stammzellen, S. 102.

35 Vgl. Ipsen (2004), S. 268 (269).

36 In diesem Sinne Schockenhoff (2003), S. 100; ebenso für eine (allerdings nicht zwangsweise durchsetzbare) Implantationspflicht Brohm (1998), Juristische Schulung, S. 197 (203). 
Person. Seine Personenwürde ist ihm als Mensch ohne Rücksicht auf seine Eigenschaften, seine Leistungen und seinen sozialen Status eigen. $\aleph^{37}$ Weiter heißt es bei Otto: Der »Idee, die Würde eines Menschen in einer vom Menschsein differenzierten Personalität zu gründen, ... liegt ein Verständnis der Person zugrunde, das nicht den Menschen schlechthin als Person erkennt, sondern nur den Menschen, der aktuelles Selbstbewußtsein besitzt. ... Mit der Idee einer unantastbaren Menschenwürde sind diese Überlegungen nicht in Einklang zu bringen, denn unantastbar ist die Menschenwürde nur, wenn sie von Anfang an mit dem Beginn des menschlichen Lebens zuerkannt wird. ... Würde knüpft an das biologische Fundament des einzelnen an, das heißt an den Beginn des individuellen menschlichen Lebens. «Dies ist jedoch keine geglückte Begründung, warum der Inhalt des Würdeprinzips der »Wert des Menschen als Menschen«, und zwar unter Einschluß des Embryonenschutzes, sein soll. Denn erstens ist unklar, warum es eine »einfache Erkenntnis« sein soll, daß das Verständnis der h.M. korrekt ist - denn eine Trivialitätsbehauptung als solche ist niemals eine Begründung. Zweitens enthalten die weiteren Ausführungen einen Zirkelschluß: Denn die Personalitätsidee widerspricht der »Unantastbarkeit«natürlich nur dann, wenn man in das Verständnis von Menschenwürde bereits das Konzept der h.M. - das doch allererst zu begründen wäre - hineinlegt. Drittens ist keineswegs ausgemacht, daß ein Abstellen auf »Personalität« wirklich einzelne Menschen vom Würdeschutz ausschließt; wir untersuchen dies am Ende des Beitrags. Viertens ist das Abstellen auf das biologische Fundament erneut eine bloße Behauptung, die als solche gerade nichts begründet und die zudem einen naturalistischen Fehlschluß enthält.

\section{Verfassungsrechtliche Position 2 - abwägbarer Schutz des Embryo in vitro:}

Bis hierher bleibt zunächst festzuhalten: Die bisherige h.M. vermag nicht hinreichend und nicht widerspruchsfrei zu begründen, warum der Embryo einen dem geborenen Menschen gleichen oder wenigstens ähnlichen Rechtsstatus haben sollte. Vielmehr schafft die h.M. mehr Probleme, als sie zu lösen vermag. Vor diesem Hintergrund sprechen manche (wenngleich nicht alle im folgenden genannten) Autoren dem Embryo den Status eines Trägers von Grundrechten und Menschenwürde gleich gänzlich ab. Diese Gegner der Anerkennung der Trägerschaft der Menschenwürde des Embryos bereits ab der Befruchtung sind gleichwohl nahezu einhellig der Ansicht, daß der Embryo gleichwohl normativ nicht irrelevant sei. Dabei sind die Konzepte zur Begründung eines Schutzkonzeptes vielfältig. Im wesentlichen stellen sie darauf ab, einen vollständigen Schutz erst ab einem bestimmten Zeitpunkt (z.B. der Nidation oder der Geburt) anzunehmen, ggf. mit im zeitlichen Verlauf steigender Intensität, während vorher eine normalerweise geringere Schutzwirkung eingreife. ${ }^{38}$ Merkel nimmt dabei

37 Vgl. Otto (2005), Juristenzeitung, S. 473 (477 f.).

38 Vgl. Dreier (2002), Zeitschrift für Rechtspolitik, S. 377 ff.; Hillmer (2004), Patientenstatus und Rechtsstatus von Frau und Fötus im Entwicklungsprozeß der Pränatalmedizin, S. 121; Enders (2003), Juristische Ausbildung, S. 666 (672); Merkel (2002), S. 268; Ipsen (2001), S. 989 (993); Kloepfer (2002), S. 417 (420 - der von einer Grundrechtsanwartschaft spricht); Schlink (2003), Der Spiegel Nr. 51, S. 51 (52); Gründel (2002), Chancen und Risiken der Gentechnologie, in: Politische Studien Nr. 1, S. 57 (68); Herdegen (2005), S. 363 ff. 
- wie z.T. auch andere - an, zugunsten des Embryos bestehe lediglich eine objektivrechtliche Schutzpflicht, und zwar eine abwägbare. ${ }^{39}$ Den Konzepten der Kritiker der h.M. ist gemeinsam, daß das wie auch immer geartete Recht bzw. die objektive Schutzpflicht zugunsten des Embryos einer Abwägung mit kollidierenden Interessen gegenübersteht (insofern nicht von vornherein das Ergebnis einer zwingenden Forschungsfreiheit feststeht). Diese Abwägbarkeit bezweifelt die h.M. demgegenüber was aber nicht ausschließt, daß das BVerfG den Schutz des Nasciturus u.U., da es die Frage offenläßt, ebenfalls als »nur objektiv-rechtlich« (nur eben als sehr stark) auffassen könnte.

Eine der im folgenden zu klärenden Fragen wird ergo sein, wie es mit der »Abwägbarkeit« der Kernprinzipien liberaler Verfassungen aussieht. Diese Frage ist schwierig, weil die Grundrechte generell sehr wohl untereinander abwägbar sind, andererseits aber das Würdeprinzip im Grundgesetz und in der EuGRC als »unantastbar« bezeichnet wird. Gleichzeitig sind gemäß Art. 1 Abs. 2 GG aber alle Menschenrechte Folge der Würde. Deshalb und weil der Fall denkbar ist, in dem die Würde des einen Menschen mit der Würde des anderen Menschen kollidier ${ }^{40}$, entsteht die schwierige Frage nach Abwägbarkeit. Merkel und andere beharren jedenfalls darauf, daß eine Abwägung zu Lasten des Embryo zugunsten der Bekämpfung schwerster Krankheiten und somit des Schutzes der Gesundheit bereits existenter Menschen generell möglich, wenn nicht sogar geboten sei. ${ }^{41}$ Allerdings steht unverändert die Frage im Raum, ob der Embryo nicht doch (womöglich von Anfang an) subjektive Rechte hat, ob diese also vielleicht doch zwingend begründbar sind. Ferner ist immer noch offen, ob sich dem Würdeprinzip vielleicht doch ein klar begründbarer Gehalt geben läßt.

\section{Eine neue Position 3 - als Teil einer allgemeinen Konzeption von Diskursrationa- lität und Generationengerechtigkeit (zugleich eine Anmerkung zum EGMR):}

Vor diesem Hintergrund soll im folgenden eine neue Position 3 formuliert werden. Sie faßt anderweitige Ausarbeitungen zu den Grundlagen der Gerechtigkeit, der Grundrechte und der Generationengerechtigkeit zusammen und wendet deren Ergebnisse sodann auf den Embryonenschutz an. ${ }^{42}$ Es handelt sich dabei um einen diskursethischen Ansatz, der allerdings gegenüber den diskursethischen Klassikern Jürgen Habermas

39 Vgl. Merkel (2002), S. 268.

40 Zur Fundierung der Menschenrechte in der Würde vgl. näher unten. Allerdings kann diese Kollision nur dann entstehen, wenn man mit Ekardt (2005), Kap. IV C. und entgegen der herrschenden Juristenmeinung in Deutschland eine Multipolarität der Freiheit (siehe wiederum unten) und zudem keinen allgemeinen Vorrang der Abwehr- vor den Schutzrechten anerkennt (oder gar die Möglichkeit von Schutzrechten insgesamt bezweifelt wie z.B. Enders (1997), Die Menschenwürde in der Verfassungsordnung).

41 Vgl. Kloepfer (2002), S. 417 (421); Merkel (2002), passim.

42 Direkt zum Embryonenschutz schon Ekardt (2004), § 5 B. IV.; Ekardt (2005), Kap. V A.; Kornack (2006), S. 260 ff.; in den ersten beiden Fundstellen findet sich auch ausführlich der nachfolgend verwendete Ansatz universaler und intergenerationeller Gerechtigkeit m.w.N. Ursprünglich ging es dem dortigen Ansatz um eine Begründung der Nachhaltigkeit (also der globalen Gerechtigkeit zwischen den Völkern und der Generationengerechtigkeit zwischen den Zeiten). 
und Karl-Otto Apel (sowie Robert Alexy, Wolfgang Kuhlmann, Dietrich Böhler u.a.) und auch gegenüber verwandten modern-liberalen Philosophen wie John Rawls in der Grundstruktur erhebliche Divergenzen aufweist. Wir beginnen mit der bisher nicht befriedigend beantworteten Frage: Warum überhaupt Menschenwürde? Nur eine Begründung dessen vermag letztlich zu klären, was Würde als zentrale Verfassungsnorm überhaupt heißen soll. Es ist darum bedeutsam, daß sich das Würdeprinzip in der Tat als zwingende Kernnorm der Gerechtigkeit ${ }^{43}$ fundieren läßt. Dies gelingt (anders als bei den altliberalen Klassikern Kant, Locke u.a.), indem man nachweist, daß das Menschenwürdeprinzip, verstanden als ein Prinzip gegenseitiger Achtung, für den Menschen als Menschen bei Strafe des Selbstwiderspruchs die unhintergehbare Basisbedingung ist, sobald man überhaupt je, und sei es auch nur manchmal, mit Gründen über normative Fragen gestritten hat, sich also normativ rational verhalten hat. ${ }^{44}$ Denn das Begründen als Vorgang impliziert logisch, daß man den Gesprächspartner als jemanden achten muß, der sich eine freie Überzeugung zu bilden vermag; andernfalls wäre das Begründen witzlos. Weil sich freilich nur ein Individuum autonome Überzeugungen zu bilden vermag und weil freies Sich-Überzeugen eine autonome Möglichkeit der Lebensgestaltung erfordert, läßt sich Würde in dieser Gedankenfolge als der gebotene Respekt vor dem autonomen Individuum auffassen, ergo als ein Prinzip, welches eine eigenständige Lebensführung für alle Vernunftwesen gleichermaßen verbürgt. Nur dies ist also der begründete Gehalt von Würde.

Die Ansicht, das Würdeprinzip meine einfach den »Wert des Menschen als Menschen«, kann darum so nicht aufrechterhalten werden. Auch die Objektformel ist von der somit gegebenen Begründung (und nicht mehr nur Behauptung) eines bestimmten Würdeinhalts nur insoweit gedeckt, als sie gegebenenfalls einen Teilaspekt des Würdeprinzips ausdrücken mag. Daß das Würdeprinzip nicht einfach den »Wert des Menschen« meint, zeigt außerdem eine Norm wie Art. 1 Abs. 2 GG (»das Deutsche Volk bekennt sich darum zu unverletzlichen und unveräußerlichen Menschenrechten ...«) oder auch die Präambel des Internationalen Paktes über bürgerliche und politische Rechte -, indem sie alle Menschenrechte in der Würde fundiert. ${ }^{45}$ Und das ergäbe wenig Sinn, wenn Würde den »Wert des Menschen als Menschen« meinte, weil dieser wohl kaum in jedem Recht steckt. Also scheint Würde etwas anderes zu sein, und zwar genau das, was der gemeinsame Kerngedanke aller Grund-/ Freiheits-/ Menschenrechte ist. Was aber ist dies? Es ist der gebotene Respekt vor der Autonomie des Individuums, welches ein Leben nach eigenen Vorstellungen und Regeln leben können soll - mit der Maßgabe, daß alle anderen diese Möglichkeit gleichermaßen haben

43 Zusammen mit dem Unparteilichkeitsprinzip, das hier nicht näher interessiert; dieses Zusammenspiel von Autonomie und Unparteilichkeit (bzw. Würde und kategorischem Imperativ) kennen alle liberalen Theorien von Kant über Rawls bis zu Habermas.

44 Rationalität ist die menschliche Befähigung, Fragen mit Gründen zu entscheiden.

45 Dies ergibt sich (entgegen gängiger Ansicht) direkt aus dem Wortlaut des Art. 1 Abs. 2 GG: Dort ist gerade nicht davon die Rede, daß sich nur »einige « Menschen- bzw. Grundrechte aus dem Würdeprinzip ergeben. Zudem zeigt sich systematisch, daß Art. 1 GG eine Art Überschrift des gesamten Menschen- bzw. Grundrechtskatalogs ist; auch deshalb können nicht nur »einige« Menschenrechte gemeint sein. 
müssen: Dies läßt sich sogar rein »verfassungspositiv« sagen. Aber auch das davor angeführte, »rechtsphilosophische« Argument kann nicht etwa so in Zweifel gezogen werden, daß man ja den Ausgangssatz »normative Aussagen (also moralische und rechtliche Sätze) müssen vernünftig sein« in Zweifel ziehen könne. Denn es läßt sich zeigen, daß der gelegentliche Vernunftgebrauch dem Menschen als Menschen unvermeidbar ist - und daß schon der gelegentliche Vernunftgebrauch den Respekt vor der Autonomie aller potentiellen Gesprächspartner und damit aller Menschen gebietet. Das Menschenwürdeprinzip und das aus beidem Ableitbare sind angesichts jener Unvermeidbarkeit (a) der Würde und (b) der Vernunft ${ }^{46}$ - die sich eben nicht auf andere Prinzipien erstreckt und damit konkurrierende Prinzipien in die Begründungslosigkeit verweist und ergo ausschließt - das, was in der liberalen Welt »Gerechtigkeit « heißt.

Daß somit die Personalität als autonomes Wesen und weniger das Menschsein als solches Gegenstand der Grundrechte ist, ist übrigens auch die kürzlich geäußerte Position des EGMR. ${ }^{47}$ Nur kann der EGMR dies erstens nicht wirklich begründen. Denn der EGMR stützt die Interpretation der Menschenrechte hier wie auch sonst zumeist auf einen Konsens der Staaten bzw. der Rechtsordnungen. Doch wer sagt, daß das, was in vielen Staaten der herrschenden Verfassungsinterpretation entspricht, deshalb per se richtig ist? Wäre es per se richtig, dann wäre die je h.M. als solche stets unkritisierbar. Zudem ist der Schluß von der faktischen Existenz bzw. Hegemonie einer Meinung auf ihre eigene Richtigkeit auch deshalb ${ }^{48}$ wenig überzeugend, weil darin wieder ein naturalistischer Fehlschluß liegt. Zweitens zieht der EGMR möglicherweise nicht die richtige Schlußfolgerung, wenn er annimmt, ein Würdeverständnis, welches an die Personalität bzw. Autonomie anknüpfe, verwehre dem Embryo eine grundrechtliche Position:

Um die Würde des Embryo kann es nach dem Gesagten nur gehen, wenn der Embryos überhaupt Träger der Grundrechte bzw. der Menschenwürde ist. Die diesbezüglich gängige Diskussion läßt aber nach wie vor hinreichende Argumente für oder gegen diese Position vermissen. Doch hat Ekardt andernorts (mindestens) ein solches Argument vorgeschlagen, welches sogar eine subjektiv-rechtliche Position von Embryonen nahelegt: Das Vernunftprinzip in normativen Fragen erwies sich bekanntlich eben als alternativlos. Alternativlos ist es aber nicht nur aus logischen Gründen, sondern auch deshalb, weil schlicht kein anderer gangbarer Weg der normativen Konfliktlösung bereitsteht. Man kann nur darüber reden; die Vernunft ist in diesem Sinne offen.

46 Diese Art des negativen Beweises kann man als (doppeltes) transzendentales Argument bezeichnen (näher dazu a.a.O.). Durch den Vorgang des negativen Beweises, der nicht positiv etwas belegt, sondern etwas als alternativlos auszeichnet, entgeht man dem sonst fälligen Einwand des Skeptikers, die Gerechtigkeit habe doch immer einen »beliebig gesetzten Ausgangspunkt« (und dann hätte die Position 3 gegenüber der h.M. nichts gewonnen). Das hat sie eben gerade nicht!

47 Vgl. EGMR, NJW 2005, 727 ff.

$48 \mathrm{Zu}$ weiteren Einwänden, insbesondere zur performativen Widersprüchlichkeit einer solchen Position sowie zu ihrer Neigung, auf neue Herausforderungen wie die Generationengerechtigkeit nicht wirklich reagieren zu können und schlimmstenfalls bis hin zu einer Rechtfertigung des Totalitarismus traditionsverbunden zu sein, vgl. im einzelnen Ekardt (2005), Kap. II A. 
Denn es ist keine Methode erkennbar, wie man ohne Argumentation (ergo mit bloß willkürlichen Behauptungen) oder aus dem Vernunftbegriff als solchem »das evident inhaltlich Richtige « als Antwort auf eine normative Frage X finden wollte. ${ }^{49}$ Auch im Verhältnis zwischen schon heute Lebenden und »künftig Lebenden« - also letztlich generell im Verhältnis der Generationen - muß man angesichts dessen sagen, daß nicht inhaltlich offenkundig ist, was hier gerecht wäre. An der damit nötigen diskursiven Klärung (etwa in einem gewaltenteiligen und demokratischen Verfahren) können sich nun aber Embryonen oder gar künftig gezeugte Menschen ${ }^{50}$ nicht beteiligen, obwohl sie von den Folgen solcher Konfliktlösungsdiskurse direkt betroffen sind. Darum ist zumindest die diskursive Konfliktlösung mit Vertretern der Zukunftsinteressen, vorliegend: der Embryonen, geboten. Die damit nötigen Verfahrensbeteiligungsrechte für Zukunftsbelange genügen aber nicht. Menschenwürde als aus dem normativen Streit mit Gründen abgeleitetes Prinzip reguliert eben nicht nur das Verfahren Gerechtigkeitsdiskurs, sondern auch die Gerechtigkeit seiner Ergebnisse; denn diese sind die Vorbedingung aller weiteren künftigen Diskurse. Besonders intergenerationell wären bloße Verfahrensrechte ohne ergebnisregulierende Prinzipien (also inhaltliche normative Prinzipien) wenig vorteilhaft, weil eben nur Vertreter die Zukunftsinteressen bzw. Embryonalinteressen benennen könnten und diese Vertreter durch die Embryonen auch nicht kontrolliert werden könnten. Die damit vernunftgebotenen inhaltlichen Anforderungen z.B. an parlamentarische »Gesetze als Diskursergebnisse (etwa das Stammzellengesetz) können Lebende gerichtlich einklagen, indem sie das Gesetz etwa auf seine Vereinbarkeit mit der Berufs- oder Forschungsfreiheit verfassungsgerichtlich überprüfen lassen. Sie können also eine Ergebniskontrolle und zugleich eine zweite, gerichtliche Diskursstufe einfordern (die nicht triviale Notwendigkeit einer Gewaltenteilung zur rationalen Lösung von Konflikten einmal vorausgesetzt). Genössen Zukunftsbelange bzw. embryonale Belange keinen Schutz als einklagbare und ergo subjektiv-rechtliche Belange - sei es auch nur durch einen Treuhänder -, wären sie hier strukturell benachteiligt. Dies aber wäre mit der offenen Vernunft ${ }^{51}$ unvereinbar.

Für Embryonen nicht zutreffend ist dagegen das ansonsten in der Generationendebatte durchaus wesentliche Potentialitäts- bzw. Ewigkeitsargument. Dieses Argument rechtfertigt den Embryonenschutz daraus, daß der Embryo das Potential habe, sich zum Menschen und damit zu einem zweifelsfreien Grundrechtsträger zu entwickeln. Ekardt bezeichnet dieses Argument als Ewigkeitsargument, weil es voraussetzt, daß

49 Ein sehr plastisches Beispiel aus der jüngsten Zeit ist hier die Empörung mehrerer islamischer Staaten wegen der karikierten Darstellung Mohammeds in der dänischen Zeitung Jyllands-Posten. Diese Staaten verweigern sich gerade einem Diskurs und rekurieren stattdessen auf »die Gefühle der Gläubigen« (vgl. nur FAZ vom 03.02.2006). Dabei wäre durchaus eine rationale Klärung der Frage möglich, wie in einer pluralistischen Gesellschaft mit unterschiedlichen Konzepten des »guten Lebens « umgegangen werden soll, wenn diese miteinander in Konflikt geraten und dadurch zu einer Gerechtigkeitsfrage werden (für die der Staat im Gegensatz zu reinen Fragen des guten Lebens gerade zuständig ist); vgl. dazu neben den Nachweisen in Fn. 1 Ekardt (2005a), Gerechtigkeit und gutes Leben in der Migrationsgesellschaft, Kritische Justiz, S. 248 ff.

50 Die im folgenden zu Embryonen gemachten Ausführungen gelten im wesentlichen für alle künftigen Menschen. 
die Grundrechte auch künftig noch gelten werden - und weil es sozusagen aus diesem künftigen Schutz ein Vereitelungsverbot in der Gegenwart herleitet, weil es ja gerade der Witz von Grundrechten ist, die Freiheit effektiv zu schützen - und dies sei bei drohenden irreversiblen Vereitelungshandlungen nicht mehr gegeben. Auf diese Weise mag man den Schutz künftiger Generationen etwa gegenüber einem globalen Klimawandel herleiten können, denn zumindest eine gewisse Zahl »potentieller« künftiger Menschen gibt es mit Sicherheit. Der Embryo in vitro als einzelner dagegen besitzt die Potentialität nur in passiver Weise, bzw. nur dann, wenn eine Pflicht bestünde, ihn bei einer Frau zu implantieren. Eine Pflicht kann aber nur bestehen, wenn es schon eine Schutzposition des Embryos gibt, die diese gebieten könnte. Insofern wird das Ewigkeits- bzw. Potentialitätsargument für zu Forschungszwecken hergestellte Embryonen gerade wieder zirkulär. Eine Implantationspflicht wäre dabei um so weniger vorstellbar, als anschließend womöglich ein Abtreibungsrecht bestünde. ${ }^{52}$

Sofern man nun allerdings »volle aktuelle Rechte « für den Embryo forderte, droht dies zu übersehen, daß der Embryo noch ungeboren ist. Es ist doch so: Die grundrechtsbegründende Menschenwürde wurzelt in der menschlichen Vernunft. Diese aber hat der Embryo noch nicht. Dies ist auch nicht wie beim Bewußtlosen oder im Koma Liegenden ein Zustand, der jede Minute enden könnte. Der Embryo ist aktuell noch kein handlungsfähiger Mensch (wenngleich er zur Gattung homo sapiens gehört). Darum genießt der Embryo richtigerweise nur eine Vorwirkung seiner subjektiven Grundrechte, die aber nicht automatisch schwächer sein muß als das Vollrecht, sofern es um irreversible Gefährdungen geht. So brauchen wir nicht länger die nicht gerade einfache (und wohl kaum definitiv begründbare) Annahme zu machen, ein winziges Lebewesen im Mutterleib sei bereits jetzt ein »Mensch « im Sinne der Grundrechte und genieße zudem »Würde«, also Respekt vor seiner Autonomie. Nicht länger müssen wir wie die deutsche Judikatur einfach behaupten, auch ein Embryo sei im Sinne des Würdeprinzips ein Mensch - wofür ja mangels Vernunft wenig spricht.

Das Menschenwürdeprinzip ist bei alledem vielleicht gar kein Grundrecht, sondern eher der Grund und damit die Auslegungsbasis der Freiheitsrechte. Den Basischarakter betont z.B. Art. 1 Abs. 2 GG explizit: Die Menschenrechte werden um der Würde willen gewährleistet (»darum«), sind also etwas von der Würde Unterscheidbares. Ferner spricht Art. 1 Abs. 3 GG von »nachfolgenden« Grundrechten, was nicht so klingt, als wäre die vorher in Abs. 1 erwähnte Würde ein solches Recht. Zudem ist die Würde gemäß Art. 1 EuGRC, 1 GG »unantastbar«. Damit kann sie aber nur einen Gehalt haben, der im Grunde niemals verletzt sein kann. Sie erscheint darum, auch wegen Art. 1

51 Offen sollte nicht mit »prozedural « verwechselt werden. Denn wir meinen anders als Habermas u.a. nicht, daß keine inhaltlichen, sondern nur Verfahrensaussagen über die Gerechtigkeit möglich wären. Unsere Ableitungen aus der offenen Vernunft haben ja »inhaltlichen « Charakter. Ebensowenig bestreiten wir (insofern übereinstimmend mit Habermas, Rawls, Kant u.a.), daß der einzelne für sein persönliches gutes Leben inhaltliche, und zwar auch »irrationale«, Orientierungen wählen darf. Dies ist sogar unvermeidlich, da persönliche Geschmacksfragen usw. nur sehr begrenzt einer rationalen Prüfung zugänglich sind.

52 Vgl. Hufen (2004), Juristenzeitung, S. 313 (318). 
Abs. 2 GG, eher als Auslegungsmaßgabe und Grund der Freiheitsrechte denn als eigenständig anwendbare - ob nun subjektiv- oder objektivrechtliche - Norm. Es würde jedenfalls wenig ändern, wenn man die Würde als Grundrecht betrachten würde, da die Auslegungsrelevanz der Würde so oder so erhalten bleibt, wie man sieht, wenn man fragt, ob der Schutzbereich der Würde beeinträchtigt ist: Ein Eingriff direkt in die Würde wäre jedenfalls nur dann denkbar, wenn der über die allgemeine Grundrechtsbegründung hinausgehende Gehalt der Würde beeinträchtigt wird. Nach der »Autonomie-Respekt-Formel « wäre demnach zu fragen, ob man die Fähigkeit des Embryos zu einer autonomen Lebensführung gänzlich in Frage stellt, indem man mit ihm forscht. ${ }^{53}$ Und es ist durchaus fraglich, ob man dies hier mehr in Frage stellt, als man es z.B. beim finalen Rettungsschuß durch Polizeibeamte in Frage stellen würde. Letztlich geht es daher wohl eher um das Recht auf Leben und Gesundheit. ${ }^{54}$

Manche halten es nun für unstimmig, dem ungeborenen Kind überhaupt etwas zuzusprechen. Hinter jener Gegenansicht steht freilich ebenso wie beim BVerfG die Vorstellung, daß derjenige, bei dem man Leben und Gesundheit schütze (sei's als Grundrecht oder als objektives Recht, wie das BVerfG u.U. im Sinn hat ${ }^{55}$ und darum einen sehr starken, aber möglicherweise nicht subjektiv-rechtlichen im Sinne von »einklagbaren« grundrechtlichen Schutz fordert), eine nahezu uneinschränkbare Position genösse. Doch kein Wortlaut irgendeiner Verfassung kennt uneinschränkbare Rechte; vielmehr werden alle Rechte stets als einschränkbar ausgewiesen. Und es kann auch niemals absolute Rechte geben, da Rechte stets untereinander kollidieren und sich dann gegenseitig beschränken müssen. Dies wird auch in der LuftSiG-Entscheidung des BVerfG übersehen. Denn Art. 1 Abs. 1 S. 2 GG zeigt (entgegen einer bisher gängigen Meinung) durch die gleichberechtigte Verwendung der Begriffe »achten « und »schützen« gerade an, daß die Würde und demnach auch ihre Ausprägungen, die Freiheitsrechte, gleichermaßen gegen direkte staatliche Intervention wie (vom Staat) gegen Gefährdungen durch die Mitmenschen zu verteidigen sind. ${ }^{56}$ Durch diese doppelte Schutzrichtung kollidieren dann z.B. die Freiheit von Lebens- und Gesundheitsbeeinträchtigungen sowie die Berufs- und Forschungsfreiheit in vielen Fällen. Zudem müßte ein absoluter Lebensschutz z.B. auch die Landesverteidigung gegen einen fundamentalistischen Angreifer schlicht unterlassen, weil sonst Soldaten in Gefahr kämen. Der »Alles-oder-nichts«-Streit, der den Embryo entweder absolut schützen oder weitestgehend ungeschützt lassen will, ist daher so nicht überzeugend - auch

53 Wobei auch diese Konstellation besser über das allgemeine Freiheits- bzw. Persönlichkeitsrecht als Grundrecht gelöst werden sollte.

54 Wenn man gleichwohl den Schutzbereich der Würde für beeinträchtigt hielte, stellte sich die Frage nach der Einschränkbarkeit der »unantastbaren« Würde. Denn selbst wenn eine Würdebeeinträchtigung vorliegt, dann bliebe zumindest eine Abwägbarkeit gegen Würdeverletzungen bei den zu heilenden Kranken denkbar.

55 Das Urteil zum Luftsicherheitsgesetz vom 15.02.06 scheint dies zu bestätigen, indem Art. 1 nur i.V.m. Art. 2 Abs. 2 GG zitiert wird.

56 Auch dazu wieder im einzelnen die Fundstellen in Fn. 1 mit weiteren und ausführlicheren Argumenten für die Multipolarität der Freiheit; direkt zur Abwehr-Schutz-Scheidung nochmals Ekardt/ Susnjar (2007), Zeitschrift für Gesetzgebung, Heft 1. 
wenn er in Deutschland z.T. üblich ist. ${ }^{57}$ Der Embryo hat vielmehr eine potentielle Vernunftnatur, kann in Zukunft ein Diskurspartner sein und genießt damit heute eine Vorwirkung des Rechts auf Leben und Gesundheit, welches wie alle Rechte aber auch Abwägungen unterliegt. Diese können auch existenzieller Natur sein.

Was ist nun in die notwendige Abwägung einzustellen? Man denke nicht nur an die Berufs- und Forschungsfreiheit, sondern je nach Fallkonstellation auch an das Persönlichkeitsrecht der Mutter oder das Recht auf Leben schwerkranker Menschen, denen durch Embryonenforschung geholfen werden könnte. All dies kollidiert offenkundig mit den Embryonenrechten. Wie aber wägt man $a b ?^{58}$ Da die »Quantität der Betroffenen « in Ermangelung besserer Gründe durchaus einmal ein Argument im Rahmen von Abwägungen sein könnte, kann es dabei durchaus sein, daß eine Argumentation für den generationenübergreifenden Menschenrechtsschutz die Erhaltung der menschlichen Lebensgrundlagen als Ganze gebietet, aber nicht die Rettung jedes einzelnen Embryos erzwingt. Jene Abwägungsnotwendigkeit scheitert allerdings auch nicht daran, daß die grundrechtliche Abwehrposition aus der Berufs- oder Forschungsfreiheit per se stärker wäre als Schutzpositionen der Embryonen oder der Kranken, sich also in der Verhältnismäßigkeitsprüfung stets durchsetze. Denn es gibt entgegen einer verbreiteten Annahme keine Regel »Abwehrrecht vor Schutzrecht«, wie wir soeben unter Verweis auf Art. 1 Abs. 1 S. 2 GG zu zeigen versuchten. Angesichts der Notwendigkeit eines differenzierten Interessenausgleichs wird man auch nicht einfach (wie es das BVerfG im LuftSiG-Urteil freilich erneut bekräftigt hat) einen absoluten Lebensschutz für »unschuldiges Leben« annehmen können.

Inhalt der Abwägung müssen dabei auch langfristige Folgen der Genforschung für den Bestand liberaler Gesellschaften, ebenso wie die möglichst weitgehende Selbstbe-

57 Die gesamte hiermit entwickelte Konzeption kann nicht etwa, wie es vielleicht Nettesheim (2005), Archiv des öffentlichen Rechts, S. 71 (95 ff.) tun würde, als »metaphysisch« und »nicht weltanschaulich neutral « zurückgewiesen werden. Es sind ja lediglich die logischen Implikationen offengelegt worden, die das Grundgesetz zwingend mit sich führt. Zudem: »Weltanschauliche Neutralität« meint in liberalen Verfassungen nicht Wertungsfreiheit. Dies ergäbe schon deshalb gar keinen Sinn, weil das Recht als Sammlung von Sollensbzw. Wertungssätzen notwendigerweise »wertend « ist. »Weltanschauungen«, die den Staat und das Recht nichts angehen, sind deshalb seit Locke und Kant vielmehr Konzeptionen des guten Lebens; und solche Konzeptionen sind in der Tat Privatsache und kein möglicher Gegenstand von Recht; dagegen sind die Grundlagen der Gerechtigkeit, um die es hier geht, zwingend mit dem Recht verbunden und gerade keine (persönlich wählbare) »Weltanschauung «. Auch wenn man stattdessen glaubt, allgemeine Gerechtigkeitssätze ließen sich nicht angeben, sondern müßten stets von der Mehrheit bestimmt werden, wäre dies im übrigen wiederum eine allgemeine Gerechtigkeitstheorie. Vgl. dazu und zur für liberal-demokratische Rechtsordnungen zentralen Scheidung von Gerechtigkeit und gutem Leben wieder die Nachweise in Fn. 1 sowie Ekardt (2005a), S. 248 ff.; Ekardt (2005b), Zeitschrift für Rechtspolitik, S. 225 ff. (wobei die Autoren dieses Aufsatzes im einzelnen darüber differieren, inwiefern genau »weltanschauliche Neutralität« geboten ist).

58 Im Detail müßte man fragen: Wie genau ist gerecht abzuwägen? Welche Verfahrensregeln sind dabei einzuhalten? Und welche Institutionen brauchen wir, damit all dies gerecht abläuft - wofür ist ein Parlament zuständig, wofür vielleicht eher ein Verfassungsgericht? Zuletzt: Welche Regeln gelten für die Erhebung entscheidungsrelevanter Tatsachen? 
stimmung (also Nicht-Objekthaftigkeit) des Menschen, sein. Hier entsteht ein schwerwiegendes Problem, welches die Abwägungsposition der Embryonen massiv stärkt und womöglich doch »ein« bestimmtes Abwägungsergebnis nahelegt: Wie wäre es, wenn es aufgrund fortgesetzter Embryonenforschung eines Tages gelänge, einen Menschen exakt zu planen? Die Frage wäre dann natürlich auch: Gibt es ein Recht darauf, gentechnisch »unmanipuliert « - oder gentechnisch »perfekt « - zur Welt zu kommen? Wichtiger wäre allerdings noch die Frage, ob nicht die liberale Ordnung als solche dann in Frage gestellt würde. Denn auch wenn die Genmanipulation die liberal-universale Gerechtigkeitsidee normativ nicht aufhebt (denn die Ableitung der Würde aus dem Vernunftgebrauch bestünde unbesehen der gentechnischen Entwicklung: auch bei »planbarer Intelligenz« könnte man in Diskursen nicht sicher sein, wer im Einzelfall das bessere Argument zur Entscheidung einer Konfliktfrage präsentiert, so daß alle Menschen weiterhin als autonome Wesen zu achten wären), so könnte doch eine weitreichende Manipulation diese Ordnung rein faktisch erodieren lassen: Wüßten die Bürger, daß einzelne von ihnen ein besonders trächtiges Intelligenzgen besäßen, andere dagegen ein »Dummheitsgen«, würde das rein faktisch ihre Achtung voreinander untergraben. Dies muß in jedem Fall verhindert werden. Denn eine liberale Ordnung möchte auch, daß sie selbst real wird; und genau dies wäre dann gefährdet. Bedacht werden muß insoweit auch, daß sich technische Entwicklungen häufig nur schwer »halb« verbieten lassen, da moderner Technik eine erhebliche Eigendynamik innewohnt.

Diese verfassungsrechtliche Position 3 bietet den Vorteil, daß sie begründen kann, warum Würde und Leben überhaupt schützenswert sind und daß sie darauf aufbauend diesen Normen einen klareren Inhalt zu geben vermag. Ferner kann die Position begründen, warum uns »künftige « Rechtsträger nicht egal sein dürfen. Damit werden auch Wertungswidersprüche zwischen »künftigen « Menschen in ihren verschiedenen Entwicklungsstadien vermieden. Dennoch sollen an dieser Stelle auch einige Schwierigkeiten dieses Ansatzes aufgezeigt werden. Mag man bestimmte generelle Einwände gegen Diskurstheorien bei genauer Prüfung durchaus zurückweisen können ${ }^{59}$, so ergibt sich gleichwohl ein spezielles, wichtiges Problem. Nimmt man als Begründungsansatz für Würde und (Grund-)Rechte im allgemeinen nicht das Menschsein an sich, sondern die (potentielle) Teilnahme am Diskurs an, stellt sich die Frage, ob hierdurch nicht eine Verengung der Berechtigten stattfindet. Was folgt daraus, wenn Ekardt feststellt »Gründe richten sich ... an jeden, der potentiell in der Lage wäre, meine Thesen zu bestreiten«? Was bedeutet dies für Menschen, die niemals die Fähigkeit haben, an irgendeiner Form des Diskurses teilzunehmen? Insofern gehen die liberalen Grund-

59 Zur Widerlegung vieler Einwände Ekardt (2005), Kap. II C.; exemplarisch für die Einwände noch Hinkmann (2002), Ethik der Menschenrechte, S. 87 ff. Insbesondere ist unsere Konzeption zwar nicht »christlich-altkantianisch«, aber auch nicht utilitaristisch: Sie ist vielmehr konsequentialistisch insofern, als sie die Folgen bestimmter Entscheidungen beleuchtet. Utilitarismus wäre demgegenüber eine Lehre, die - anders als wir vorliegend normative Fragen für nicht mit Gründen entscheidbar hält und deshalb eine Verrechnung der rein faktischen Bürgerpräferenzen vornimmt. Und weder diese Verrechnung noch das Ausgehen von rein faktischen Präferenzen ist unsere Position. 
prinzipien als Ausfluß der »logischen Implikationen der Kategorie Grund « formal betrachtet vielleicht doch nicht »von einer Kategorie aus, die allen Menschen als Menschen gemeinsam ist $\ll{ }^{60}$ Müßte man dann gar annehmen, vor einer Implantation eines in vitro fertilisierten Embryo sei zunächst verpflichtend diagnostisch sicherzustellen, $\mathrm{da} ß$ dieser auch potentieller Diskursteilnehmer ist?

Dieser prinzipielle Einwand ist auch gegenüber klassischen Diskurstheorien formuliert worden. Für das vorliegende Thema ist er von besonderem Interesse. Zwar läßt sich das Problem reduzieren, indem man auf dreierlei hinweist: Erstens kann auch bei vermeintlich »dummen « Menschen nie ausgeschlossen werden, daß sie im konkreten Fall wesentliche Gründe zu präsentieren in der Lage sind (so daß die Rechtsstellung des Menschen gerade nicht von seinem erwartbaren Diskursbeitrag abhängig gemacht werden kann). Zweitens ist eine allgemeine »Theorie der Dummheit« als solche kaum formulierbar. Drittens kann selbst bei Geisteskrankheiten nie ganz ausgeschlossen werden, daß sie durch den medizinischen Fortschritt eines Tages heilbar sein werden. Es mag aber gleichwohl eine (allerdings sehr kleine) Gruppe von Menschen zurückbleiben, die z.B. mit derart schweren Hirnschäden auf die Welt gekommen sind bzw. zu kommen drohen, daß man nahe daran ist, auch für die Zukunft ihre Diskursfähigkeit »sicher« auszuschließen.

Auch dieses Problem läßt sich aber möglicherweise dadurch argumentativ lösen, daß eine Beachtlichkeit nicht am Diskurs Beteiligbarer dadurch erreicht wird, daß deren Beachtlichkeit als Bedingung der Freiheitlichkeit einer Gesellschaft insgesamt gesehen würde. ${ }^{61}$ Auf diese Weise würde man einen indirekten Freiheitsschutz der erwähnten Schwerstbehinderten über die Freiheitsrechte der Mitmenschen herleiten. Der Gedanke dabei ist: Eine Gesellschaft, die in bestimmter Weise mit Vertretern der Gattung homo sapiens umgeht, droht in einer Weise zu verrohen, die letzten Endes auf den Umgang der diskursfähigen Menschen untereinander überzugreifen droht. Man könnte jetzt allerdings zum einen die damit gestellte empirische Prognose in Frage stellen. Zum anderen könnte jemand finden, dies stelle den Eigenwert der Schwerstbehinderten in Frage. Doch dies ist falsch: Erstens setzt dieser Einwand wieder den alten Würdebegriff der h.M. voraus. Zweitens steht zu vermuten, daß der Fall, daß wir für die Zukunft eine Diskursfähigkeit definitiv ausschließen können, aus erkenntnistheoretischen Gründen niemals auftreten kann, da man prinzipiell nie bestimmte medizinische Verbesserungen ausschließen kann. Drittens begründet die vorliegende Position doch gerade einen Wert aller Menschen (was u.E. der h.M. bisher nicht gelungen ist), nur eben auf eher indirektem Wege. Letztlich sehen wir hier aber weiteren Klärungsbedarf.

60 Alle Zitate bei Ekardt (2004), S. 65 (Hervorhebungen im Original); zur hier formulierten Kritik schon Hösle (1997), Die Krise der Gegenwart und die Verantwortung der Philosophie, 3. Aufl., S. 265 ff.; zur Antwort darauf z. T. schon Ekardt (2005), Kap. II C.

61 Argumentiert man hier anders, besteht die Gefahr, daß man genau den eigentlichen Vorteil der Diskursethik einbüßt - indem man nämlich das Problem durch Rückgriff auf Werte, die nicht ihrerseits diskursimmanent sind, zu lösen versucht. Dies passiert etwa bei Habermas (2001), Die Zukunft der menschlichen Natur, passim. 
Das Beispiel der Embryonenforschung zeigt sehr deutlich, daß es kaum möglich ist, unbeschränkbare Rechte zu gewährleisten, ohne sich dabei an irgendeiner Stelle in Wertungswidersprüche zu verwickeln. Insofern kommt man im Bereich pränatalen Lebens zu einem in sich stimmigeren Konzeptes, wenn man von einer Vorwirkung von Grundrechten ausgeht. Es bleibt daher zu hoffen, daß sich der parlamentarische Gesetzgeber und die Verfassungsgerichtsbarkeit der Sache noch einmal zuwenden, die entstandenen Widersprüchlichkeiten auflösen und sich auch der Frage des Inhaltes und der Begründung der Menschenwürde noch einmal vertiefend widmen. Aber die Diskussion um die Embryonenforschung wird auch über die nationale Ebene hinaus weiterzuführen sein. ${ }^{62}$ Wünschenswert wäre hier auch eine verstärkte Zusammenarbeit im Sinne eines globalen Stammzellprojektes, um zu einheitlichen Standards zu gelangen, was auch die Frage der Patentierbarkeit im Bereich der Biomedizin einschließen müßte. Um allerdings eine internationale Zusammenarbeit zu ermöglichen, bedarf es einer universalistischen Konzeption der Gerechtigkeit. Denn nur sie kann in ihren Grundprinzipien unabhängig von temporären oder lokalen gesellschaftlichen Anschauungen sein und damit weltweite Anerkennungsfähigkeit beanspruchen. Da der Embryonenschutz weitgehend leerliefe, wenn die Forschung usw. schlicht in andere Länder ausweichen kann, sind die Grundprinzipien des Embryonenkonflikts bei alledem letztlich ein Fall für eine globale Politikebene.

$62 \mathrm{Zu}$ den national unterschiedlichen Bestimmungen vgl. nur die Länderberichte bei Taupitz (2002), Das Menschenrechtsübereinkommen zur Biomedizin des Europarates - taugliches Vorbild für eine weltweite Regelung? 